\title{
FINITE ELEMENT ANALYSIS OF THIN BERYLLIUM WINDOWS FOR A MUON COOLING CHANNEL
}

\author{
J. N. Corlett, N. Hartman, D. Li; \\ Lawrence Berkeley National Laboratory, Berkeley, CA 94720, USA
}

\section{Abstract}

The cooling channel for a muon collider or neutrino factory may utilize thin beryllium windows to separate RF cells and enhance on-axis accelerating fields. The nominal windows for an $805 \mathrm{MHz}$ design are composed of $16 \mathrm{~cm}$ diameter circular foils, 127 microns thick. These windows undergo significant ohmic heating from RF power, and displace out of plane, causing the cavities to detune. In order to understand how to control this effect and to evaluate different window designs, a Finite Element Analysis (FEA) model was created in ANSYS, and this model was correlated to windows tested in the laboratory [1]. The prototype windows are actually brazed assemblies, and the thin foils in these assemblies become pre-stressed during cool down from braze temperature, complicating the analysis. Using empirically validated models, several other window designs are analyzed, such as windows of different thicknesses, non-constant thickness windows ("stepped" designs), and windows of diameters much larger than $16 \mathrm{~cm}$.

\section{INTRODUCTION}

One design of RF cavities (the so-called "pillbox") being considered for the muon cooling channel in either a muon collider or neutrino factory contains windows of 127 micron thick foils of high purity IF1 beryllium. For the muon collider proposal, these windows are situated in cavities which operate at $800 \mathrm{MHz}$, with a foil radius of $16 \mathrm{~cm}$; for the neutrino factory, on the other hand, windows operating at $200 \mathrm{MHz}$ with diameters of $38 \mathrm{~cm}$ or more are being considered. Each foil is brazed between two annular rings of lower purity PS200 beryllium, with annuli of $16 \mathrm{~mm}$ and $40 \mathrm{~mm}$ for the 800 $\mathrm{MHz}$ and $200 \mathrm{MHz}$ windows respectively; the rings are each approximately $1.6 \mathrm{~mm}$ thick (Figure 1).

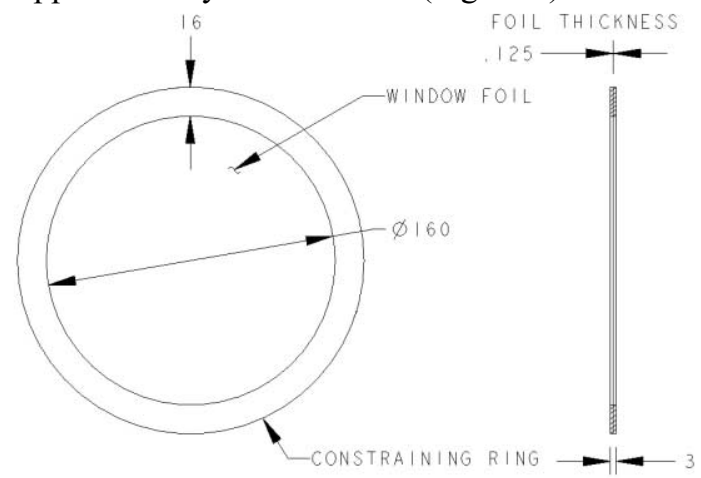

Figure 1. Layout of Beryllium test window (all dimensions in $\mathrm{mm}$ ).
The windows displace due to ohmic heating, and these displacements must be kept below 25 microns in order to minimize the cavity's frequency shift to below $10 \mathrm{kHz}$ [2]. The goal of this research is to develop a computational model which allows us to determine window deflections under different power loads and thicknesses, to compare this model with experimental results, and to apply the model to possible design solutions.

A complication of the window's assembly, however, is that the rings and foil are made from materials with different coefficients of thermal expansion (CTE). These differing CTEs induce pre-stress in the window during cool down from the brazing process. The pre-stressed model is very sensitive to the CTEs of the materials and how these properties vary with temperature. Also, very little data is available on the properties of high purity IF1 versus lower purity PS200 Beryllium. The temperature at which brazing occurs is also only approximately known. In order to account for these uncertainties and to build a reliable model of the pre-stressed window, the model had to be created, compared to experiment, fiducialized, and then re-checked. This iterative process was used to arrive at a "best fit" model for design purposes.

\section{COMPARISON OF BASELINE MODELS TO EMPIRICAL DATA}

In order to generate empirical data, the prototype beryllium window was illuminated with a halogen bulb set to varying voltage, while the temperature distribution on the window was measured with thermocouples and the displacement at center was measured with a dial gauge. The measured temperature distributions were then input into the ANSYS FEA model, the deflection was calculated, and the CTE properties were manipulated in order to create agreement between the model's deflections and the empirically measured results. Since the window's displacement tolerance is approximately 25 microns, the final model was adjusted to be most accurate at small deflections (Figure 2) [3].

In addition, the CTE was modeled as both varying and constant with temperature. Due to the multitude of confounding factors in calculating the displacements, only approximate agreement was obtained, and this was found to be most accurate when the CTE was assumed constant with temperature. Although this approximation is nonphysical, it provided a more predictive model for design purposes (Figure 2). 


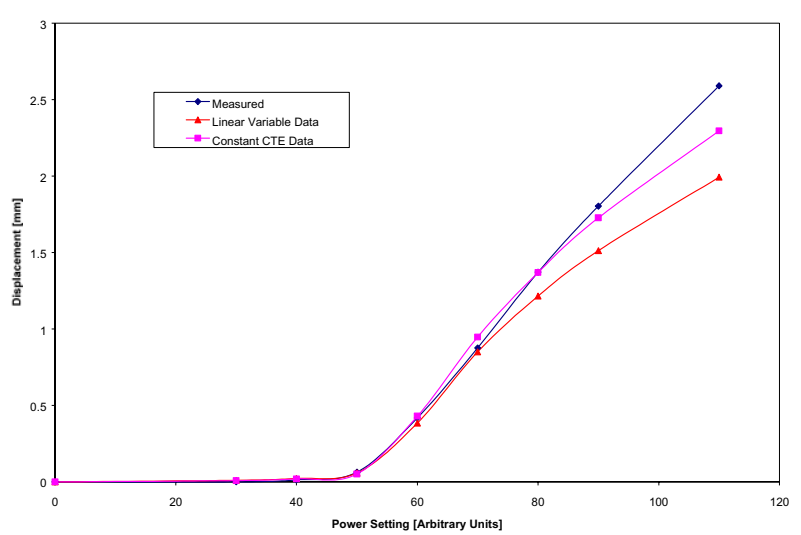

Figure 2. Maximum displacement as a function of power dissipated in halogen heating bulb, for two FEA models and empirical test model.

\section{REAL WORLD LOADS VERSUS FINITE ELEMENT LOADS}

The empirical displacement data collected for this analysis was driven by heating from a halogen bulb, which produces a parabolic temperature distribution in the Beryllium foil $[4,5]$. RF heating, however, produces a temperature distribution that is flatter than parabolic, as shown in Figure 3. To allow for comparison, the models created in ANSYS were loaded with parabolic temperature distributions corresponding to the measured distributions in the majority of experimental cases. The displacement produced in a window is dominated by the temperature rise in the window (difference between maximum and minimum temperatures); the shape of the temperature profile is secondary, but not inconsequential. Thus, while examining the model's response to known parabolic temperature distributions is useful for comparison, it must be analyzed with the expected RF temperature profile in order to judge whether it passes the 25 micron tolerance under real world conditions.

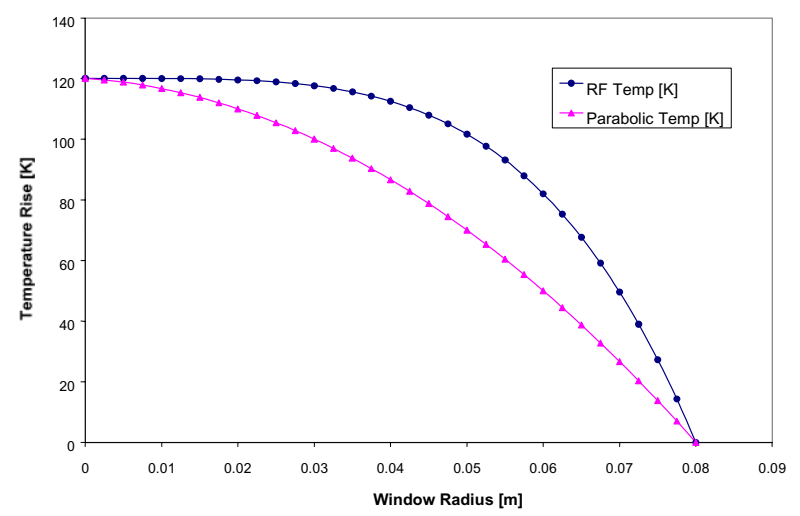

Figure 3. Different temperature profiles with the same overall dT.

The baseline model, shown in Figure 1, was analyzed with both RF and parabolic temperature loads of different magnitude temperature rise. From this baseline, the allowable temperature rise in the window was calculated and can be used as a benchmark for other design variations (Figure 4).

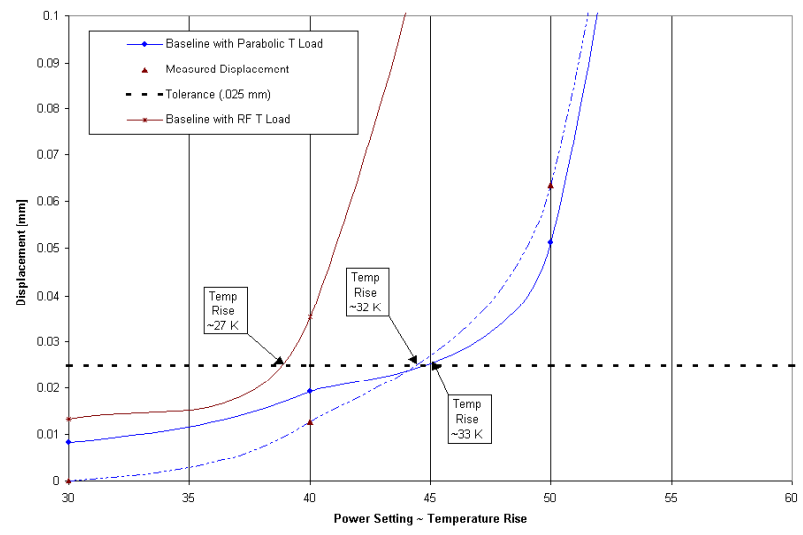

Figure 4. Close-up of 25 micron tolerance regime for baseline model under RF and Parabolic temperature loads.

\section{WINDOW DESIGN PARAMETERS}

\subsection{Windows of Different Thicknesses}

The baseline model results suggest that the allowable temperature rise for this window is less than $27 \mathrm{~K}$. Since the expected temperature rise in normal operation is anticipated to be more than $35 \mathrm{~K}$, some development is necessary in order to arrive at a working window design. The most obvious modification is increasing the window's thickness. This increase will result in two effects: increasing the thermal conductance in the window and increasing the window's stiffness. If the window behaves primarily as a membrane, then the stiffness will increase linearly with thickness; however, if the window experiences significant bending, then the stiffness may increase by as much as the cube of the thickness. The thermal conductance, however, scales with the window's thickness, so in order to compare similar power dissipations on windows of different thicknesses, it is necessary to scale the temperature loads by the inverse of the thickness ratios.

For the purposes of this comparison, we analyzed models with three thicknesses: the standard 127 micron (.0050") window, a 190.5 micron (.0075") window, and a 254 micron (.0100") window. Figure 5 shows displacement results for models analyzed with both parabolic and RF (scaled to match maximum temperature rise) temperature distributions. As expected, the RF power distributions caused displacements significantly larger than their parabolic counterparts.

Table 1. Safe operating temperature rises for windows of different thicknesses.

\begin{tabular}{|c|c|c|}
\hline $\begin{array}{c}\text { Window T } \\
\text { (microns) }\end{array}$ & $\begin{array}{c}\text { Safe Temp. under } \\
\text { RF Load }\end{array}$ & $\begin{array}{c}\text { Safe Temp. under } \\
\text { Parabolic Load }\end{array}$ \\
\hline 127 & 27.1 & 33.1 \\
\hline 190.5 & 27.3 & 33.9 \\
\hline 254 & 29.9 & 36.8 \\
\hline
\end{tabular}




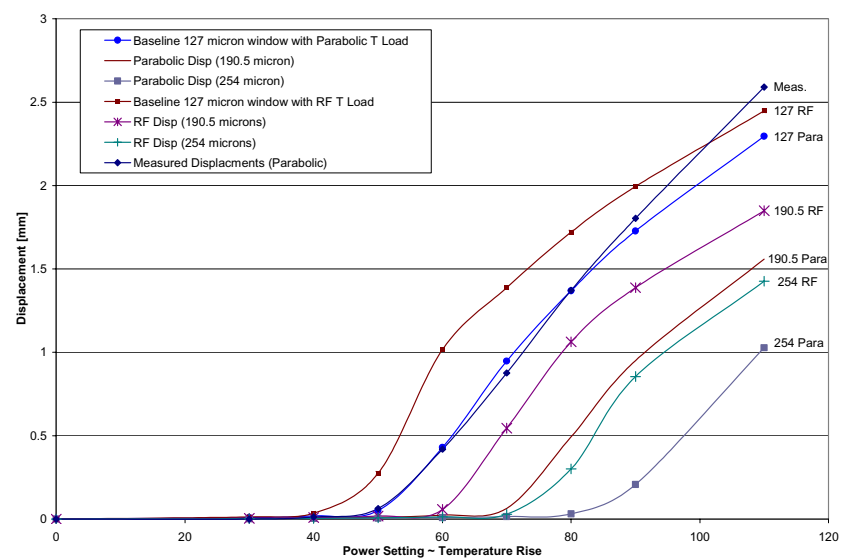

Figure 5. Displacement vs. power setting curves for windows of different thicknesses under RF and parabolic temperature distributions.

Examination of the displacement data allows us to calculate the temperature rises at which the window deflects by the tolerance of 25 microns. These "safe" temperature rises for both the parabolic and RF load cases are shown in Table 1. It is noticeable that the safe temperature is relatively constant for all of the window cases. This implies that the window acts almost entirely as a membrane - since thermal elongation in the plane of the window causes membrane-type behavior, and planar elongation depends only on temperature rise. Although the allowable temperature rises remain the same, the thicker windows can withstand proportionately more power input before reaching the limit.

\section{2 "Stepped" Window Designs}

While thickening the window has been shown to increase the power dissipated before exceeding the deflection tolerance, it carries with it the disadvantage of increasing muon scattering. This effect is most noticeable at low radius where the muon beam is most dense, and becomes less important at the outer extents of the window. To make use of this effect, we developed a model of a "stepped" window, which is thin in the center, but thicker at the outer radii. For the purposes of this investigation, it was assumed that the inner thickness would be 127 microns, while the outer thickness would be 254 microns. The step transition radius (the point at which the foil thickens) was varied from $20 \mathrm{~mm}$ to 60 $\mathrm{mm}$. In addition, the window was modeled with a onesided step, asymmetric about its thickness.

For the stepped window models, only RF temperature distributions were used. The RF temperature profiles computed previously were scaled to account for the thicker sections of the stepped windows. In this way, the total power dissipated in the window is held constant, as compared to other models with RF temperature loads of the same "power setting." Adding a step as small as 20 $\mathrm{mm}$ wide (i.e. at $60 \mathrm{~mm}$ radius) significantly improves the thermal performance. As the step radius decreases, the relative advantage of the step becomes smaller (Figure 6).

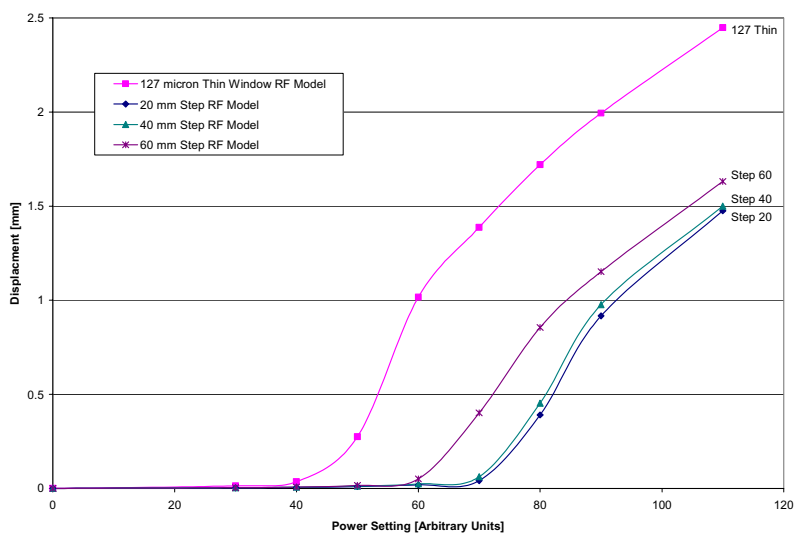

Figure 6. Displacement vs. power setting curves for step windows of different step radii under RF temp. loads.

\subsection{Windows Larger than $16 \mathrm{~cm}$}

Up to this point, only $800 \mathrm{MHz}$ windows have been examined. The same ANSYS model, however, was changed to model $200 \mathrm{MHz}$ windows of three possible diameters: $38 \mathrm{~cm}$ ID, $42 \mathrm{~cm}$ ID, and $46 \mathrm{~cm}$ ID. The larger windows were also modeled with the same thickness variations explored in the $800 \mathrm{MHz}$ design: $127,190.5$, and 254 microns. The $200 \mathrm{MHz}$ models were subjected to RF power loads that were scaled to apply total power dissipations in the range of 0 to 200 Watts. In general, displacements were very large, and radius changes produced comparable percentage changes in overall displacement. The anticipated power dissipation during use is approximately 120 Watts, which corresponds to at least $6 \mathrm{~mm}$ of displacement. The maximum power that any of the analyzed windows can dissipate without moving more than 25 microns is approximately 40 Watts, which corresponds to a temperature rise of $34 \mathrm{~K}$ across the window. Interestingly, this temperature rise is slightly higher than the corresponding "safe" temperature rise found for the $800 \mathrm{MHz}$ window ( $28 \mathrm{~K})$.

\section{CONCLUSIONS}

These results encourage further development of $800 \mathrm{MHz}$ window designs, in particular prototyping and testing in an RF test cavity. However, they also show that for $200 \mathrm{MHz}$ operation, significant design changes will be necessary for windows to operate within tolerance.

\section{REFERENCES}

[1] ANSYS is a trademark of SAS Inc. www.ansys.com [2] D. Li, et al., "Be Window Studies at Room Temperature", MUCOOL 110.

[3] N. Hartman, et al., "Thin Beryllium Windows Analysis and Design Status ", MUCOOL 180.

[4] J. Corlett, et al., "Deflection measurements of thin foils for the muon cooling channel RF cavities", MUCOOL 62.

[5] N. Hartman, et al., "Comparison of Deflection Measurements to FEA Modeling for Muon Cooling Channel RF Cavity Windows", MUCOOL 111. 